University and Chile's NationProject. Readings of an architectural narrative

\section{| RESUMEN |}

El presente es un ensayo acerca del fenómeno arquitectónico que sucede en La Alameda frente a la Casa Central de la Universidad de Chile. Los autores sostienen que, debido a su contenido histórico y valor simbólico, la arquitectura de ese lugar comunica las ideas y voluntades principales que han convergido en la construcción de Chile como país. La lectura comienza al contrastar una imagen antigua con el estado actual del sector y preguntarse por los motivos de los cambios.

\section{| ABSTRACT |}

The present essay is about the architectural phenomenon that occurs in Alameda in front of the institutional building of the University of Chile. The authors argue that, because of its historical significance and symbolic value, the architecture of the place communicates the main ideas and willingness that have converged in the construction of Chile as a nation. The reading begins by contrasting an old image with the current state of this area and wonders about the reasons behind the changes.

ANDRÉS WEIL P.* + WERÓNICA WEIL P.**

\title{
La Universidad y el Proyecto-País Chile. Lecturas de un relato arquitectónico ${ }^{* * *}$
}

\section{LA ARQUITECTURA COMO RELATO COSMOLÓGICO}

El fenómeno arquitectónico corresponde a todas las acciones humanas que han convergido, directa e indirectamente, en la materialización de la arquitectura. Es un proceso que comenzó cuando el ser humano adquirió noción del tiempo y con ello, la conciencia de su mortalidad. Frente a esta angustia y como un medio para trascender a la muerte, el hombre, inventa la arquitectura. Desde entonces, la especie humana ha construido en sus edificaciones un relato de su cosmovisión y registrado las relaciones de poder que existen al interior de sus sociedades. A diferencia de los relatos verbales, que utilizan signos y símbolos abstractos para mediar la realidad, la arquitectura capta directamente el entorno y le da forma. Es así como en ella se materializa una conversación entre vivos y muertos acerca del significado del mundo.
La arquitectura es filosofía hecha piedra. Su fundamento está en el verbo, uno tal que es capaz de convocar a todos los actores necesarios para conspirar en su favor y convertirlo en materia. En cuanto verbo concreto, la arquitectura es un fenómeno colectivo que se organiza en acciones políticas que dan forma a la polis y transforman a sus habitantes en sujetos de derecho (ciudadanos). En este proceso, las ciudades se han erigido en narraciones concretas, relatando en piedra los valores que cohesionan a quienes las construyen y habitan.

A diferencia de las teorías científicas que se remiten a un campo meramente especulativo, los proyectos de arquitectura se comprueban en su concreción y uso. Un proyecto es una teoría acerca del sentido de habitar. Una idea inicial es seguida por un proceso de diseño que, basado en el ensayo y error, culmina en la construcción de un espacio habitable. A través de su uso en el

* Arquitecto Universidad de Chile (1985), estudios de Posgrado Universidad de Stuttgart, Alemania. Académico, Facultad de Arquitectura y Urbanismo Universidad de Chile. Profesor Asistente. Cátedra: Taller de arquitectura y epistemología.

** Odontóloga U, de Chile 1981, Académico Facultad de Odontología Universidad de Chile, Instructor, Cátedra: Bioética. Alumna del programa de Doctorado en Filosofía. Universidad de Chile, Facultad de Filosofía y Humanidades.

*** Título ponencia presentada al congreso de filosofía "La idea de universidad" Santiago de Chile, mayo de 2014 
1. Postal de Santiago. Circuló en las décadas de 1930 y 1940. Fuente: http://www.fund-edllb.org/postales.htm, autor desconocido.

tiempo, la teoría implícita en un proyecto se va validando, proceso que puede extenderse hasta su ruina ${ }^{[1]}$ y olvido ${ }^{[2]}$

El fenómeno arquitectónico es multidimensional: Su entendimiento requiere relacionar la obra -el texto-, su utilidad -el pretexto-, su entorno físico-temporal -el contexto-y su significado trascendente-el metatexto- (Philips \& González, 2009).

Sobre la base de las definiciones anteriores, se propone leer el relato que nos ofrece el fenómeno arquitectónico "Alameda Universidad de Chile" y preguntarnos, por el sentido que tiene lo universitario en nuestro país. El ejercicio se realizará a partir de una imagen captada en la principal avenida de Santiago en 1930.

\section{EL RETRATO DE LA REPÚBLICA}

Las postales buscan retratar los países. Por medio de la síntesis artística de un instante, comunican lo esencial del lugar. Es el caso de esta hermosa postal de Santiago que circuló en las décadas de 1930 y 1940. En primer plano se observa La Alameda de las Delicias, el lugar de encuentro social más importante de la época. Bernardo $O^{\prime}$ Higgins, su impulsor y cuyo nombre lleva esta avenida, denominó el proyecto "Campo de la Libertad Civil"[3]. A través de esta obra, quien fuera el primer jefe del estado chileno, buscó comunicar y hacer trascender los ideales por los cuales había luchado en vida. En la foto destacan las asoleadas fachadas de la acera sur de La Alameda que, a pesar de tener una arquitectura diversa, forman un conjunto armónico en el que sobresalen, la Iglesia de San Francisco y la Casa Central de la Universidad de Chile. Como telón de fondo se alza la imponente cordillera nevada, el milenario cordón geográfico que caracteriza el

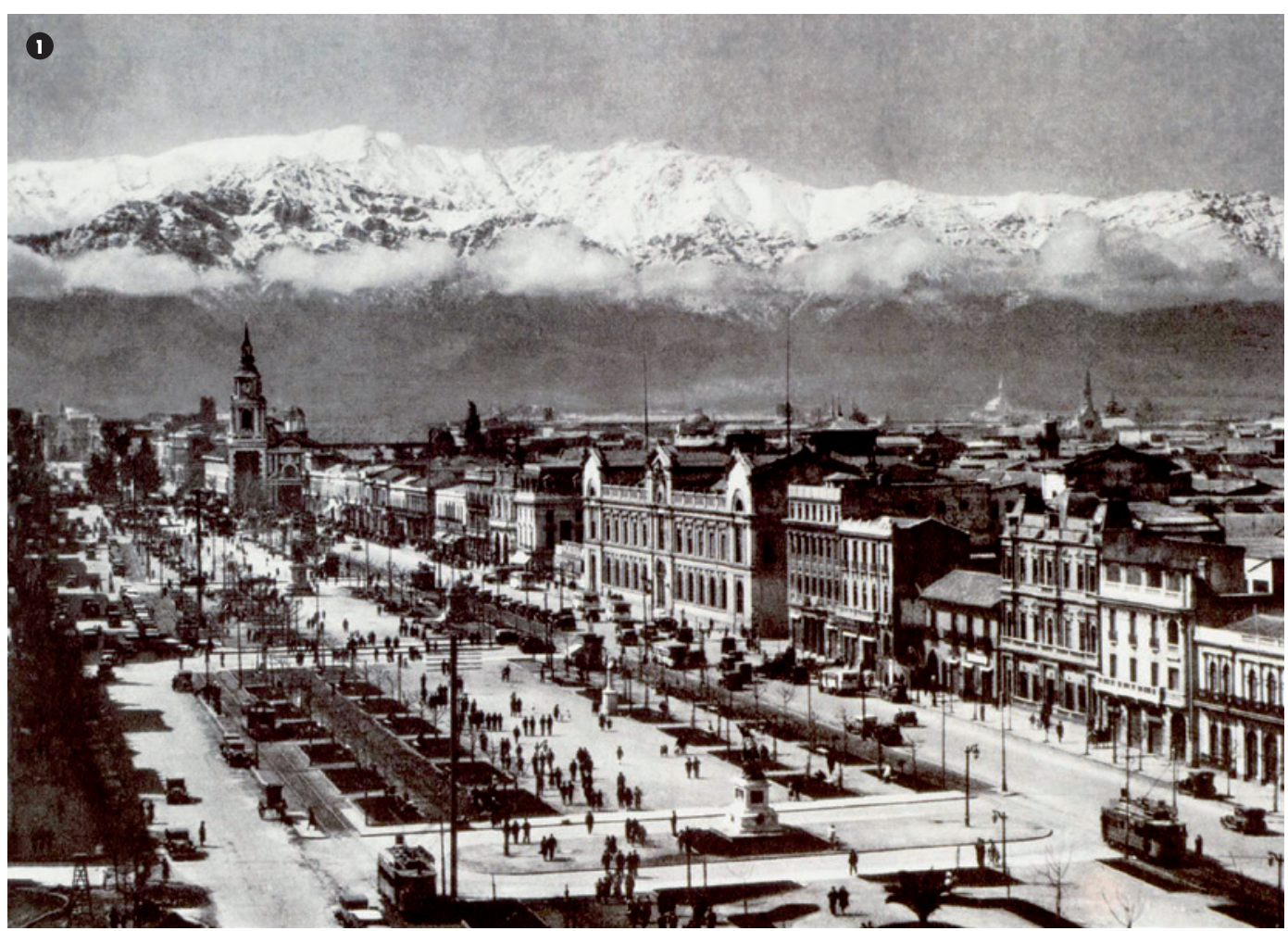

paisaje natural de nuestro país. En este retrato, la cordillera representa lo "arquitectónico", el principio original a partir del cual se ordena el cosmos explicado por la tradición mapuche ${ }^{[4]}$.

\section{La Alameda está construida sobre el lecho} del río Mapocho, curso fluvial que nace en el glaciar del cerro El Plomo. Esta montaña, la más alta de la cuenca de Santiago, era sagrada para los antiguos habitantes del valle. En ese lugar se junta la tierra con el cielo, allí convive lo humano y lo divino. Las aguas, que bajan en sentido oriente-poniente, siguen la trayectoria solar. Al hacer una lectura sutil de la situación retratada se puede decir que, la avenida principal de Chile corre sobre un haz de energía vital que une la cordillera con el mar, el día con la noche y la vida con la muerte.

Hitler hizo diseñar obras de arquitectura para que fueran hermosas ruinas.

2] Mientras no olvidemos La Atlantis, esta seguirá existiendo como teoría y fenómeno arquitectónico.

Es la denominación que figura en el decreto que instruye su construcción.

El pueblo originario del territorio central de Chile.

De las edificaciones que destacan en la foto, la Iglesia de San Francisco es la más antigua. Frente a ella comenzaba el Paseo de las Delicias que albergaba la vieja Pérgola de San Francisco. La posición de la nave central, desaplomada respecto de las otras construcciones que enfrentan La Alameda, hace que destaque por encima de las demás. El claustro de la Iglesia es un oasis de paz y tranquilidad en la mitad del ajetreo urbano, un verdadero milagro que permite entrar en comunión con la naturaleza. Estas características han hecho que el templo de San Francisco se convierta en el símbolo arquitectónico más conocido y querido de la ciudad.

El otro edificio que destaca en la foto es la Casa Central de la Universidad de Chile. La construcción está emplazada entre Arturo Prat y San Diego, al centro del tramo fundacional del paseo De Las Delicias. Es una zona de gran contenido simbólico, justamente en el lugar donde La Alameda es cruzada por el eje vial Independencia-Gran Avenida: La primera 
corresponde al brazo sur del río Mapocho, el cauce por donde bajaban las aguas de la montaña sagrada; el segundo corresponde al camino del Inca, la vía por donde transitaban los mortales (Heidegger, 2001) de norte a sur para habitar el territorio. Se trata de una ubicación emblemática: la convergencia de lo sagrado con lo humano que remite el origen del relato arquitectónico Universidad de Chile, a tiempos inmemoriales.

La morfología del edificio, un volumen extendido con fachada principal hacia La Alameda, devela el domicilio de una institución volcada a lo público, lo que en la postal está representado por el magnífico espacio ciudadano que antecede la construcción. Este retrato de Chile nos muestra una Universidad albergada en el corazón de la nación, ocupando un rol gravitante en el proyecto-país representado por La Alameda.

La Universidad de Chile está unida desde su origen al destino de la República. En 1813, cinco años antes de la creación de la Academia Militar, don José Miguel Carrera sienta sus bases al fundar el Instituto Nacional. El estratega de la Independencia entendió desde un principio que la viabilidad de Chile como país soberano, requería no solo de un ejército que custodiara sus fronteras sino que, antes de eso, de una institución que promoviera la idea de país. Tres décadas después, el Estado de Chile encarga a don Andrés Bello la redacción del Código Civil y la creación de la Universidad de Chile. Ambos proyectos se relacionan y forman parte del conjunto de elementos que definen el diseño institucional de la República. El código civil tenía por objetivo hacer viable la justicia en Chile, objetivo que solo sería posible si se creaba además, una institución pública para el cultivo de la verdad y de las virtudes humanas a través del saber y el arte.

En 1930, La Alameda de Las Delicias y la Universidad de Chile eran motivo de orgullo nacional. El tradicional paseo capitalino era el principal punto de encuentro social: allí se celebraba el Dieciocho de Septiembre, la Navidad y el Año Nuevo. El escritor Carlos
Franz (2001), en un ensayo sobre la identidad nacional, concluye que La Alameda de Las Delicias representa el mito de la Ciudad de los Césares, un lugar del imaginario colectivo chileno donde todos convivimos felices en igualdad y riqueza. Así lo entendía también el presidente Salvador Allende cuando, en su dramático discurso de despedida, hace mención a las amplias alamedas.

Con motivo de la celebración del centenario en 1942, la Universidad llamó a un concurso para crear el himno. La letra de la composición ganadora señala una universidad mucho más amplia que la actual, conformada por sus "egresados, maestros y estudiantes", es decir, por una comunidad universitaria comprometida con el pensar y el hacer país. En esa época por ejemplo, nadie cuestionaba el modelo de financiamiento de la Universidad con fondos estatales. Se entendía que era una institución pública altamente rentable para el Estado, el que recuperaba su inversión a través del aporte directo e indirecto que los propios egresados hacían a las arcas fiscales.

A partir de la lectura arquitectónica de la fotografía se puede concluir que la postal retrata a un país agradecido de su territorio y de su organización política, que ve confiado el futuro iluminado por una universidad albergada en el corazón de sus ciudadanos.

\section{LA NACION FRENTE AL ESPEJO}

La IMAgen 2 está publicada en Wikipedia bajo el concepto Av. del Libertador Bernardo O'Higgins. Fue captada, desde un ángulo similar, setenta y siete años después que la postal. Lo primero que destaca de ella es su encuadre vertical que revela el cambio que, en la percepción humana del espacio, ha sufrido el lugar. La Alameda de las Delicias retratada en la postal parece haber desaparecido y con ello dejado en el olvido el proyecto-país que alguna vez representó. Los ciudadanos fueron desplazados del "campo de la libertad civil" por vehículos particulares y de locomoción colectiva que circulan en frenéticas carreras contra el tiempo, situación que recuerda a las hordas de Atila arrasando el foro romano.

En la acera sur de La Alameda, con excepción de la Iglesia de San Francisco y la Casa Central de la Universidad de Chile, todas las demás construcciones han sido reemplazadas por edificios de variados estilos modernos que carecen de la unidad formal y la armonía estética que tenían los antiguos. Por otro lado, el aire sucio y contaminado de la capital, las edificaciones en altura y la imposibilidad de pasear por el centro de la avenida, han hecho que desaparezca la presencia de la Cordillera. Las veredas están invadidas por caóticos paraderos de buses donde se forman largas filas de espera hasta altas horas de la noche.

El domingo 20 de abril de 2014, el diario El Mercurio de Santiago anunciaba en su portada la próxima reapertura de la Casa Central de la Universidad de Chile. El histórico edificio, que fue clausurado al público después del terremoto de 2010, aún tenía vestigios de los daños que le había provocado el terremoto anterior, en 1985. La noticia señalaba que los trabajos se habían llevado a cabo gracias a la donación de un banco español, ya que durante todos estos años, el Estado nunca había considerado los fondos necesarios para su recuperación. El artículo consigna tres décadas de indiferencia pública por la institución que representa el alma máter, el espíritu de la nación chilena.

Unos meses antes, la tercera sala de la Corte Suprema acogió un recurso de protección presentado por el párroco de la lglesia de San Francisco. La resolución judicial obliga al Municipio de Santiago a responder por los daños que, durante la noche, grupos antisistema le hacen a nuestro monumento histórico. Producto de las transformaciones que ha sufrido La Alameda, el tejido urbano se ha desarticulado a lo largo de ella generando espacios abandonados, en pleno corazón capitalino, que son ocupados por tribus urbanas que se manifiestan a través del grafiti. Esta situación tiene superada la capacidad de reacción de las autoridades. 
2. Fotografía Av. Libertador General B.O'Higgins. Fuente: Santiago Puro Chile es tu cielo azulado, autor: Enrique Faúndez de Santiago de Chile.

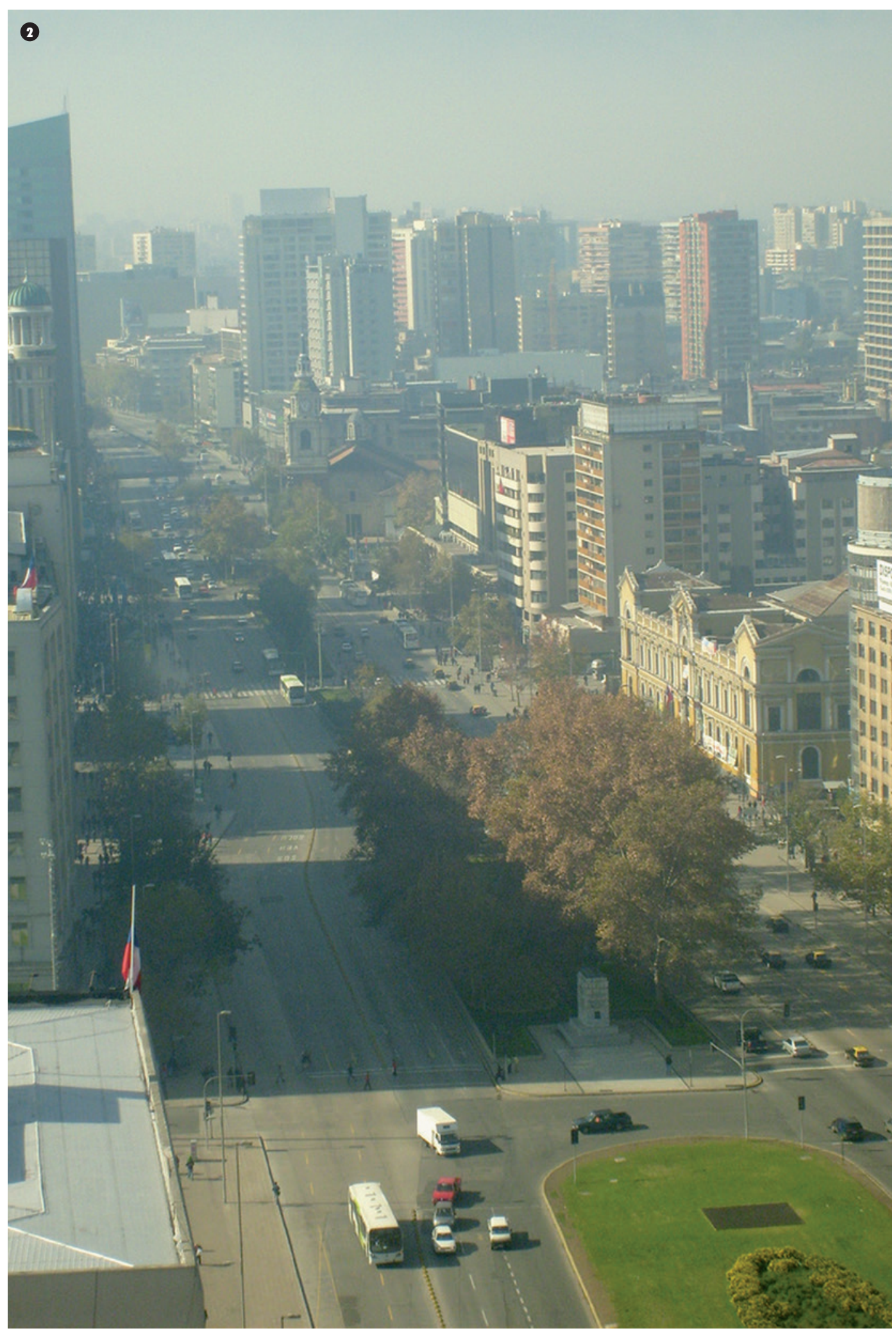

De la lectura arquitectónica de La Alameda actual se puede concluir que, en los últimos sesenta años, el sueño de la República se ha convertido en una verdadera pesadilla. ¿̇ué fue lo que sucedió? ¿Cómo fue que llegamos a la situación actual? Trataremos de dar algunas luces a estas preguntas a partir de la arquitectura, el testigo insobornable de la historia.

\section{CHILE, UN PROYECTO CONGELADO POR LA GUERRA FRÍA}

Cuando la Universidad de Chile celebró su centenario en 1942, la Segunda Guerra Mundial se encontraba en pleno desarrollo. El enfrentamiento bélico finalizó tres años después. Sin embargo, el conflicto ideológico entre capitalismo liberal y comunismo, continuó hasta 1989 en la forma de una guerra fría. Por más de cuatro décadas, las naciones vencedoras compitieron por imponer en el mundo sus respectivos modelos de desarrollo. Nuestro país no estuvo ajeno al conflicto. Testigo de aquello son las transformaciones que sufrieron La Alameda y la Universidad.

En 1949, con el propósito de ampliar las calzadas de circulación vehicular, las autoridades decretan la desaparición de la Pérgola de San Francisco ubicada en el sector central de La Alameda. En esa época se aprobó la Ley de Defensa de la Democracia, conocida como la ley maldita, que permitió la persecución por motivos ideológicos en nuestro país. Estos dos hechos podrían consignarse como los primeros signos visibles de la Guerra Fría en Chile.

En los años siguientes, el paseo por el centro de La Alameda se fue achicando para dar más espacio al automóvil, el ícono cultural del estilo de vida que los EE.UU. impondría en el mundo. La historia de las floristas de La Alameda inspiró la comedia musical más taquillera de Chile, LA Pérgola de LAS Flores, estrenada en 1960. Su éxito puede entenderse en gran medida por el dolor que, en el imaginario colectivo, provocó la pérdida del país retratado en la antigua postal. 
La Guerra Fría también se libró en el campo de la arquitectura con el triunfo del Racionalismo. Esta tendencia, conocida como Movimiento Moderno o Estilo Internacional, afectó la identidad de muchas ciudades en todo el mundo. El fundamento epistemológico del Racionalismo, es la suposición que el proceso de diseño arquitectónico es sistematizable científicamente. La idea resultaba altamente atractiva en un escenario de disputa ideológica, donde ambos oponentes compartían una fe ciega en la ciencia. No es de extrañarse entonces que el Estilo Internacional, con su receta formal abstracta de placa y torre, se impusiera en ambos lados de la Cortina de Hierro. Varios casos de ese estilo subsisten en La Alameda. Por ejemplo el Edificio Copacabana, construido al frente de la antigua pérgola de San Francisco, destaca por su singular placa de estacionamientos en cinco niveles; también lo hace el edificio ubicado en Alameda con Arturo Prat, cuyo diseño se apegó fielmente a la fórmula placa-torre, una construcción que expresa su desarraigo al lugar a través del deterioro congénito que transmite al entorno.

Durante la década de 1950 y dentro del marco del conflicto ideológico mundial, la planificación urbana toma una gran importancia en nuestro país. En 1960 se aprueba el primer Plan Regulador Metropolitano de Santiago, el instrumento jurídico que hizo posible dotar a la ciudad de una estructura vial metropolitana. El Plan permitió desarrollar un ambicioso proyecto de infraestructura urbana, entre cuyos logros está la construcción de la avenida Norte-Sur, la circunvalación Américo Vespucio y el ferrocarril metropolitano de Santiago. El proyecto del Metro consideraba la recuperación del parque central de La Alameda ya que, al acomodar el transporte público masivo en el subsuelo, se dispondría de espacio suficiente para reconstruir el tradicional paseo.

El conflicto ideológico impactó muy fuerte en la Universidad. Las reflexiones críticas se transformaron en eslogan a favor o en contra de los modelos en conflicto. Por esa vía su misión original, el cultivo de la verdad y de las virtudes a través del saber y el arte, se fue olvidando. Al final se impuso la idea que el ingreso masivo a la universidad resolvería los problemas sociales y económicos del país. Esa fue la principal motivación para llevar adelante la reforma universitaria a fines de los años sesenta y sigue siendo el gran argumento para justificar la proliferación de universidades privadas en la actualidad.

La Guerra Fría tuvo un desenlace trágico en Chile. El golpe militar de 1973 quebrantó el orden constitucional. Le siguió una dictadura que duró 17 años. El régimen de facto cambió por completo la política universitaria y la de planificación urbana. La Universidad de Chile fue intervenida por rectores militares y el Plan Regulador de 1960 quedó sin efecto por un decreto dictado en 1977.

Cuando se produce el Golpe de Estado, los trabajos del Metro estaban en plena ejecución. Se trataba del proyecto urbano más ambicioso de la historia de Chile. Encarnaba la política de integración y modernidad social que los partidos políticos de la época decían promover. Después de la inauguración del primer tramo en 1975 el trazado de la línea que iba por La Alameda fue modificado por las nuevas autoridades. El sueño social de Chile representado en la obra del Metro, fue desviado por la avenida "Once de Septiembre", en dirección a la "Escuela Militar". En 1980, junto con la aprobación de la nueva Constitución, la obra llegó a su destino y el proyecto fue detenido.

Las amplias alamedas se transformaron en pistas de carrera para buses de empresas particulares que competían por pasajeros sobre el flamante Metro. De un modo concreto se implementó en La Alameda el modelo de la libre competencia, el mismo que sirvió de referencia para crear el actual sistema de financiamiento universitario.

La Constitución de 1980 hizo viable el surgimiento de una multiplicidad de establecimientos privados que podrían competirle "el mercado de la educación superior" a las universidades tradicionales. Ese fue, probablemente, el mayor logro ideológico de la dictadura: la idea de universidad fue asimilada a la de educación superior. Dos conceptos diferentes comenzaron a ser manejados como sinónimos: educación y universidad.

En Chile, el sistema educativo distingue entre: Educación Básica, que entrega a niños los conocimientos y normas fundamentales para su desenvolvimiento como futuro ciudadano, y la Enseñanza Media, que permite el aprendizaje de materias conocidas a jóvenes que preparan su futuro profesional. A la universidad se puede acceder cuando se es ciudadano con plenitud de derechos y obligaciones: la institución universitaria es para individuos adultos que deseen formarse en el campo infinito del conocimiento universal.

Al hablar de educación para adultos, como es el caso de la educación superior, se está significando "reeducación" o "adoctrinamiento", algo muy extendido en regímenes totalitarios. Cabe destacar que si la esencia universitaria implica un compromiso con la verdad, el adoctrinamiento sería contrario a su espíritu.

Con el fin de adaptar el funcionamiento de la universidad al modelo económico impuesto por la dictadura, fue necesario desvirtuar su labor. El concepto de educación superior permite concebir su acción como un bien transable en el mercado, no así la idea de universidad como lugar donde se cultiva la verdad porque, la verdad no le pertenece a nadie en particular lo que impide ponerle precio.

Cuando se restablece el orden democrático en Chile, la misión universitaria estaba desnaturalizada. De esa forma, los vencedores del conflicto ideológico lograron que el país no volviera a pensarse a sí mismo y tuviera que sobrevivir alimentado por ideas foráneas.

La Alameda de las Delicias y la Universidad de Chile retratadas en 1930, apelan al sueño del país libre y soberano que los fundadores de la República nos heredaron. Las transformaciones que ambas sufrieron en los últimos 65 años surgieron en el contexto de la Guerra Fría. La arquitectura de Santiago dejó registro del 
3. Esquema ordenamiento de los tranvías en La Alameda de las Delicias.

4. Esquema análogo propuesto para el Andén Metropolitano.

enfrentamiento intelectual que en 1989 terminó por imponer, en todo el mundo, el modelo cosmológico de dominación de los vencedores. Desde entonces, el proyecto país Chile se ha ido desvaneciendo del ideario nacional.

\section{UN PROYECTO PARA LA ALAMEDA Y LA UNIVERSIDAD}

Los relatos arquitectónicos corresponden a los mensajes que sus creadores dejan de un modo tácito o explícito en la arquitectura. Su comprensión emerge cuando existe la voluntad de involucrarse en el fenómeno arquitectónico lo que sucede, por ejemplo, al encargar un proyecto específico (Weil, González \& Phillips, $2011)$. Con ese objetivo el TIA $A^{[5]}$ formula los trabajos académicos a sus alumnos. Fue así como el año 2012 les encargó un proyecto para La Alameda, el lugar donde el año anterior, ellos mismos, habían marchado en demanda por cambios en el sistema educativo: los protagonistas del movimiento estudiantil debían reflexionar acerca de su experiencia ciudadana a través de una propuesta de arquitectura.

El proceso de diseño se inició con un diagnóstico: las amplias alamedas se han convertido en estrechos bandejones viales; el tramo fundacional del Paseo de las Delicias, entre la Iglesia de San Francisco y Teatinos, ha desparecido; al poniente subsiste sumido en la decadencia y el olvido producto del aislamiento que crean las vías sobredimensionadas por donde circulan buses medio vacíos. El espacio urbano que ha simbolizado históricamente el proyecto país, está sitiado por el tránsito vehicular, evidenciando así las relaciones de poder que imperan en la sociedad.

A partir de ese diagnóstico se planteó recuperar el tradicional paseo capitalino (Weil, 2013). Para ello se propone reducir las actuales diez vías de circulación a tres por cada lado: dos pegadas a

[5] Se refiere al Taller que el autor dirige en la Facultad de Arquitectura y Urbanismo en la Universidad de Chile.
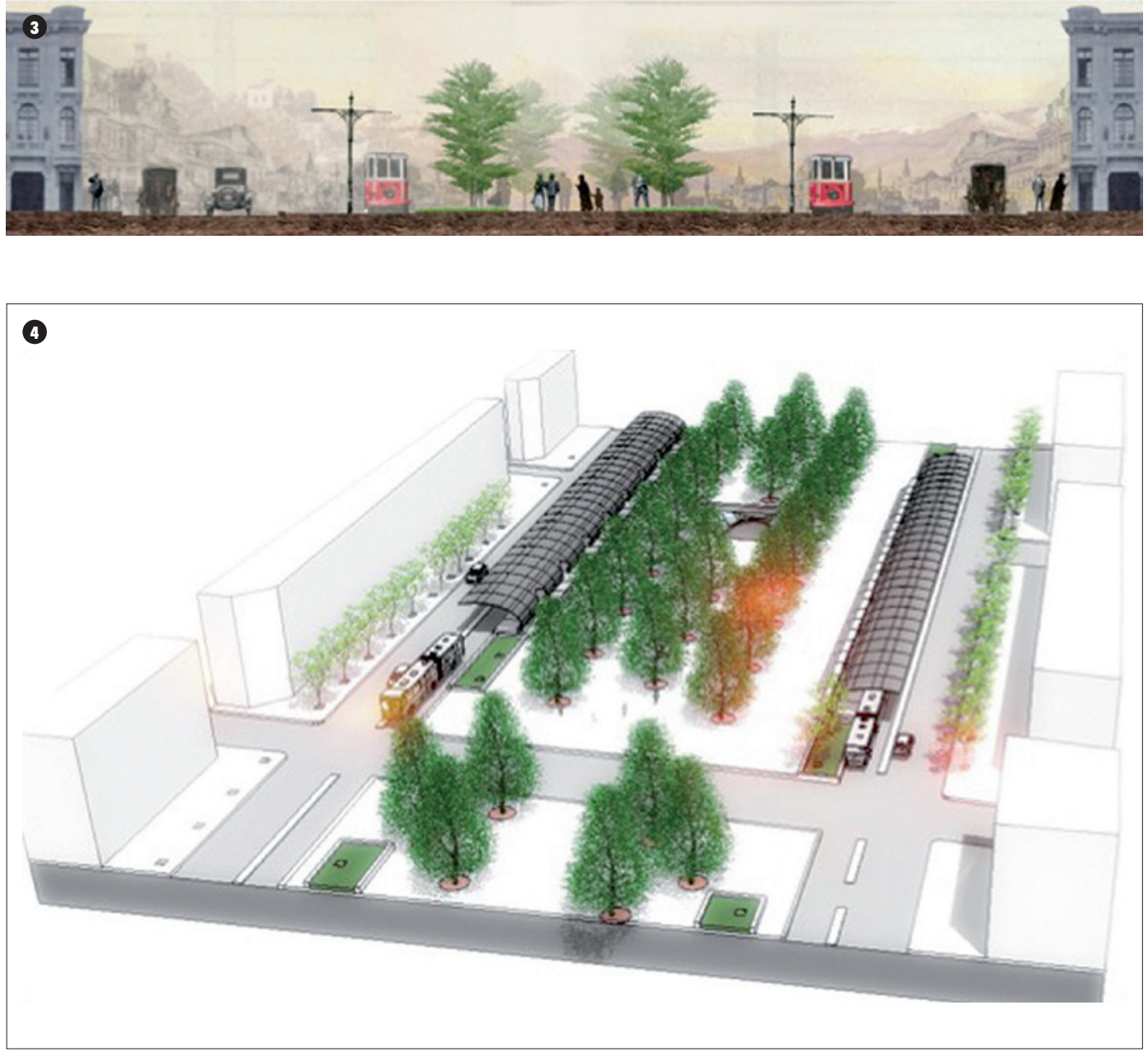

la vereda para el tránsito de vehículos livianos y una junto al bandejón central exclusiva para buses. La solución considera la operación con las nuevas máquinas del Transantiago que incorporan puertas al lado izquierdo. De esa manera se reconstruiría La Alameda de Las Delicias en la forma de un gran "anden metropolitano", un hall de recepción ciudadano, un espacio de aceptación mutua (Maturana, 1990) en el corazón de la República que acogería a todos en igualdad y dignidad.

La propuesta permitiría resolver además una serie de problemas relacionados como, por ejemplo: impedir la evasión del pago de pasaje en buses, regularizar la periodicidad del servicio, mejorar el confort de los pasajeros, establecer una conexión directa entre el paseo y el Metro, aumentar la seguridad vial, permitir los virajes de automóviles a la derecha, disminuir el vandalismos nocturno y recuperar las veredas para el comercio, la recreación y el turismo.

En cuanto a las demandas estudiantiles, se pensó en un rediseño conceptual de la Universidad: los egresados deberían ser incorporados a la comunidad universitaria cómo garantes de su autonomía económica y política, relacionando el financiamiento de la institución con los impuestos que pagan al fisco y, como una señal del compromiso de la Universidad con el proyecto-país que a diario construyen. En términos institucionales, esto implicaría incorporar a los egresados al claustro elector de 
Rector y Decanos, además de garantizar su justa representación en los Consejos de Facultad y en el Senado Universitario.

\section{SÍNTESIS: LA IDEA DE UNIVERSIDAD EN CHILE}

En Chile, la idea de universidad ha estado asociada históricamente al proyecto de país. Su origen está indisolublemente asociado al diseño institucional de la República. Lo anterior queda expresado en la ubicación y características arquitectónicas del edificio de la Casa Central de la Universidad de Chile, que son probablemente únicas en el mundo. ¿̇En qué otro país, la universidad tiene un emplazamiento urbano tan relevante como en Chile? Tal es la importancia estratégica de la Universidad de Chile, que durante la Guerra Fría se convirtió en el blanco encubierto de quienes se disputaban el poder. Los vencedores del conflicto ideológico han sido exitosos al lograr imponer la idea de la libre competencia en el quehacer universitario. La competencia es incompatible con la misión de la universidad porque genera desconfianza, la desconfianza promueve el engaño y el engaño corroe el principio de verdad. El conflicto ideológico del siglo XX se resolvió en Chile desvirtuando la idea de universidad e imponiendo a cambio el concepto educación superior como bien de consumo.

La Universidad de Chile, el alma máter, se creó para desarrollar el espíritu de la nación sobre la base de valores republicanos. Las universidades estatales son las únicas instituciones públicas a la que se ingresa exclusivamente por méritos académicos. La obligación que se desprende de ese privilegio, es un auténtico compromiso con la verdad. La condición de profesional universitario tiene implicancias éticas fundamentales para el orden político de la República. Cuarenta años de debilitamiento universitario en Chile, ha tenido como secuela el descrédito moral de muchas de las instituciones del país.

La comunidad universitaria, formada por sus egresados, maestros y estudiantes, heredaron una obligación con el destino de la nación. Muchos de los actuales dirigentes políticos, entre ellos la propia presidenta de la República, estudiaron gratis en la Universidad. ¿̇Se habrán preguntado alguna vez por qué? Cabe señalar, que ellos pudieron hacerlo porque otros, antes que ellos, tuvieron el sueño de un país libre y soberano, donde imperaría el derecho y la justicia, donde todos sus hijos podrían desarrollar sus potencialidades y ser felices. Dirigentes políticos y autoridades universitarias, que en su momento estudiaron sin la necesidad de endeudarse o endeudar a su propia familia, tienen hoy el deber moral de reponer el proyecto-país que les fue encomendado.

La Alameda de las Delicias, retratada en la postal, desapareció. Sin embargo, su esencia arquitectónica no lo ha hecho. Actualmente el relato material de La Alameda y de la Casa Central de la Universidad de Chile permiten comunicarnos con nuestros antepasados, de quienes heredamos un país independiente, cuyos ciudadanos tienen derecho a participar en la construcción de la verdad a través de la institucionalidad universitaria, pudiendo acceder a ella gratuitamente sobre la base de aptitudes académicas demostradas en una prueba anónima.
La recuperación de la Alameda de las Delicias es factible desde un punto de vista económico, técnico y de eficiencia urbana. Reconstruir nuestro campo de la libertad civil, podría ser la manifestación de una voluntad real de abrir las grandes alamedas en Chile. Lo que falta es el coraje para cuestionar los prejuicios ideológicos impuestos por la Guerra Fría. Mientras no hagamos ese ejercicio seriamente al interior de la Universidad, Chile carecerá de un proyecto que haga viable su desarrollo como país.

\section{BIBLIOGRAFÍA}

Franz, C. (2001). La muralla enterrada (Santiago, ciudad imaginaria). Santiago: Planeta.

González, J., Phillips, P. (2009). Arquitectura, Identidad, Ficción. Seminario Investigación, Carrera de Arquitectura, Prof. Guía Andrés Weil. Santiago: Facultad de Arquitectura y Urbanismo, Universidad de Chile.

Heidegger, M. (2001). "Construir, habitar, pensar" en Conferencias y artículos. Traducción de Eustaquio Barjau. Barcelona: Ediciones del Serbal.

Maturana, H. (1990). Emociones y lenguajes en educación política. Santiago: Centro de Estudios del Desarrollo.

Weil, A. González, J., Phillips, P. (2011). "Ficciones arquitectónicas: Un medio para comunicar identidad" en Revista Sociedad \& Equidad N ${ }^{\circ}$ 2. Santiago: Facultades de Ciencias Sociales, Filosofía y Humanidades Universidad de Chile. Weil, A. (2013). Lo que relata La Alameda. Documento inédito, Taller Weil, Facultad de Arquitectura y Urbanismo, Universidad de Chile. 